\title{
Effects of Age and Gender during Three Lingual Tasks on Peak Lingual Pressures in Healthy Adults
}

\author{
Elizabeth Rachel Oommen'1, Youngsun Kim² \\ ${ }^{1}$ Department of Speech Pathology and Audiology, Calvin College, Grand Rapids, Ml; ${ }^{2}$ Ohio University, Athens, OH, United States
}

Purpose: This study examined the effects of age and gender during three intra-oral lingual tasks (elevation, protrusion, and depression) on peak lingual pressure in healthy adults.

\begin{abstract}
Methods: Healthy adults were divided into groups based on age, young (18 to 35 years) and older (>60 years) adults, and gender. All the 49 participants completed maximum isometric pressure intraoral lingual tasks (elevation, protrusion, and depression) and peak anterior lingual pressures were recorded using the three-bulb tongue array of the Digital Swallowing Workstation $^{\mathrm{TM}}$. Peak lingual strength was recorded as the average of peak lingual pressures of the three trials for each lingual task. A three-way ANOVA, where the independent variables were age, gender, and lingual task, was conducted to determine the effects on the dependent variable, peak lingual pressure.
\end{abstract}

Results: Younger adults were found to exhibit significantly greater peak lingual pressure when compared to older healthy individuals, while no differences in peak lingual pressures were observed between men and women. Among the three intraoral lingual tasks, peak lingual pressures were highest during lingual depression and least during lingual protrusion.

Conclusions: The effects of age, gender, and lingual task on peak anterior lingual pressure, and the applicability of such lingual tasks in lingual strengthening exercises are discussed.

Keywords: Age, Gender, Lingual task, Maximum isometric pressure, Lingual strength

\section{INTRODUCTION}

Lingual function is imperative for important physiological events during oropharyngeal swallowing such as bolus formation and manipulation [1-7]. Contraction of the intrinsic and extrinsic muscles of the tongue [3], in addition to that of the suprahyoid muscles, contribute to functional lingual movements in speech and swallowing $[8,9]$. Impaired lingual function could result in dysphagia, manifestations of which may include difficulties in mastication, incomplete bolus formation, difficulties in bolus positioning, increased residue, impairments in oral and pharyngeal bolus transit, and premature spillage $[4,6,10,11]$. Objective values that reflect lingual strength, defined as the "capability of the muscle fibers to produce or resist force" [8, p. 658], include peak swallowing pressure, peak lingual pressure during isometric lingual tasks, and percentage of maximum tongue pressure $[1,6,12]$. Isometric lingual tasks involve maintaining a static posture of the tongue against resistance, during which the lingual pressure generated can be measured, free of changes in muscle length $[8,13]$.

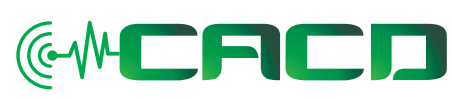

Received: February 10, 2018

Revision: August 25, 2018

Accepted: August 30, 2018

\section{Correspondence:}

Elizabeth Rachel Oommen

Department of Speech Pathology and Audiology, Calvin College, 1810 East Beltline Av. SE, Grand Rapids, MI 49546, United States

Tel: +616-526-6016

Fax: +616-526-6601

E-mail: eo23@calvin.edu

(C) 2018 The Korean Association of SpeechLanguage Pathologists

This is an Open Access article distributed under the terms of the Creative Commons Attribution NonCommercial License (http://creativecommons.org/ licenses/by-nc/4.0/) which permits unrestricted noncommercial use, distribution, and reproduction in any medium, provided the original work is properly cited. 
Examining and comparing lingual tasks that involve the anterior tongue, in an effort to document differential effect of intraoral lingual tasks on peak lingual strength, would be the basis for development of specialized lingual strengthening exercise programs in populations with dysphagia. Most often, lingual tasks involving the anterior tongue are adopted in lingual strengthening exercises (LSE), as the anterior tongue is capable of generating greater pressures during swallowing and isometric tasks when compared to other regions of the tongue $[14,15]$. Specifically, LSE in healthy individuals and in patients with dysphagia have typically incorporated anterior lingual elevation tasks [8,12,16-21]. However, LSE involving other lingual tasks such as lingual protrusion and lateralization have been explored, and such tasks were observed to result in greater strength gains in lingual pressure than those involving lingual elevation [22]. In addition, estimating tongue strength with a combination of lingual tasks may be a better estimate of oral dysphagia than when individual tasks are used [1]. This study proposes to examine the effects of maximum isometric pressure (MIP) lingual tasks involving intraoral protrusion and depression, in addition to elevation, to assess differential effects of lingual tasks on peak lingual pressures, which would add to the body of literature in this area. The lingual tasks in this study reflect lingual movements that are or can be adopted clinically in LSE.

Reduced tongue strength in older healthy individuals has been attributed to age-related changes such as reduced muscle mass, which in turn may influence swallowing functions $[5,23]$. Robbins et al. [5] found that older healthy adults ( $>60$ years) exhibited reduced maximum isometric tongue pressures than younger and middle-aged groups. Although reduced peak lingual strength during maximum isometric lingual tasks have been recorded in older healthy subjects [4-6, 14,24-27], similar peak swallowing pressures have been recorded in both groups [5-7,24]. However, the percentage of maximum pressure during swallowing was greater for the older group, indicative of a reduced "pressure reserve" [5, p. M261]. In addition to examining anterior lingual strength during maximum isometric lingual elevation tasks, Clark and Solomon [27] examined the effects of age on lingual tasks such as lingual protrusion and lateralization, and older individuals exhibited significantly reduced pressures, when compared to young and middle-aged healthy subjects, in these tasks as well.

Mixed findings have been reported when examining the effect of gender on maximum lingual strength. Even though some studies have established that men were found to exhibit greater anterior maximum isometric lingual pressure than women $[6,11,14,15,25,28]$, others have reported of no significant differences in peak anterior lingual strength between men and women $[7,24,27]$. Thus, this study aims to determine the effects the effects of age and gender during three lingual tasks (intraoral elevation, protrusion, and depression) on peak lingual pressure in healthy adults.

\section{METHODS}

\section{Participants}

All the 49 participants who completed the protocol were healthy adults with oral structure and function within normal limits, no history of dysphagia, no neurologic and head and neck impairments that affected swallowing, and had not smoked for at least five years. Individuals were excluded from participating if they reported oral sensory or olfaction deficits and gastrointestinal disorders or any treatments that impacted their swallowing ability. The young age group consisted of 13 men $(M=24.23$ years $)$ and 12 women $(M=21.83$ years), ranging from 18 to 35 years $(M=23.04$ years, $S D=4.06)$. The older group consisted of 12 men $(M=68.25$ years) and 12 women $(M=69.46$ years), ranging from 60 to 83 years $(M=$ 69.46 years, $S D=6.97)$. Flyers with general information about the study and the inclusion criteria were send by email and mail to university groups (departments, students, and faculty) and local community sites. Interested individuals contacted the researcher, who scheduled sessions in the research lab. Participants were compensated for their time and effort.

\section{Instrumentation}

Lingual pressures were recorded using a three-bulb array from KayElemetrics $^{\circledR}$, connected to the Digital Swallowing Workstation $\left(\mathrm{DSW}^{\mathrm{TM}}\right)$. The array consisted of three air-filled bulbs connected to a metal spine. Each of the three air-filled bulbs are 13 $\mathrm{mm}$ in diameter and are placed on a silica strip $8 \mathrm{~mm}$ apart [12]. The silica strip is attached to a stainless-steel spine, which extends for $12 \mathrm{~cm}$. Thin tubing emerges from each of the airfilled bulbs and attaches to the connector, which is plugged into the box that constitutes the hardware module [29], which in turn was connected to the DSW ${ }^{\mathrm{TM}}$. The bulbs were calibrated prior to recording lingual pressures, and a sampling rate of $1,000 \mathrm{~Hz}$ was used was used for the protocol. Graphical displays of lingual pressure were displayed on the DSW ${ }^{\mathrm{TM}}$. 


\section{Procedure}

Participant screening was completed after receiving informed consent from the participants. Screening measures involved having the participants complete a questionnaire and a brief oral motor examination. The questionnaire consisted of open ended and polar questions, and was designed to collect demographic information, and confirm general health status and swallowing function of participants. None of the participants reported any significant medical and neurological history, and dysphagia. Four participants reported using permanent upper bridges; however, this did not interfere with the placement of the tongue bulb array and completion of the tasks. Oral motor tasks designed to assess labial, lingual, and palatal structure and function were completed. Minimal amounts of thin liquid (water) were given to the participants to assess swallowing function, and no overt signs of dysphagia were observed.

The placement of the lingual bulb array varied depending on the lingual task. For the lingual elevation task, the bulb array was placed on the midline of the hard palate in such a manner that the anterior bulb was located at the alveolar ridge $[5,12]$. The bulb array was placed in the oral cavity by the investigator and was maintained in position by using the spine of the array. A mark was placed on the spine of the array $7 \mathrm{~cm}$ from the bulb end and this mark was consistently positioned anteriorly to the participant's closed lips to ensure consistency [15]. For two participants, only two bulbs were placed inside the oral cavity as placement of all three bulbs resulted in discomfort. Therefore, for these participants, the middle and posterior bulb was placed at the site of the anterior and middle bulb, respectively. For the lingual depression task, the furthest bulb from the spine of the array was placed at the junction between the lower incisors and gums. During this task, the participant was asked to exert pressure against the specified bulb that was supported by the mandible. Again, the researcher placed and maintained the position of the tongue array during the trials. For the lingual protrusion task, the bulb array was positioned against a tongue depressor kept between the participant's upper and lower incisors [22,27]. All the participants were able to tolerate the positioning of the bulb array for the lingual protrusion and depression tasks.

To ensure minimal activity of the jaw closing muscles, a bite block ( $2 \mathrm{~mm}$ high) was kept in between the upper and lower molars if the masseter activation, which was observed with surface electromyography (sEMG), exceeded that of baseline activity [30]. The participants were instructed to relax their jaw muscles during the lingual tasks, and the average activation of the masseter was observed to be as less than $4.5 \mu \mathrm{V}$ across all participants. For each lingual task, practice trials were completed, after which three trials each extending for 4 seconds were completed. Brief intervals of rest were provided between trials and verbal encouragement was given to the participants throughout the trials to exert maximum pressure [22,27].

For the MIP lingual elevation task, the participants were shown the anterior bulb against which maximum tongue pressure was to be exerted, which was the first bulb of the array closest to front their teeth. The following instructions were given to each participant: "Place your tongue tip on the first tongue bulb. When I say go, push your tongue as hard as you can against the first bulb. Continue to do so until I say stop," $[27,31]$. For lingual protrusion and depression tasks, the participants were shown the specific bulb against which maximum lingual pressure was to be exerted on, and the instructions were similar to that provided during lingual elevation. Counterbalancing of tasks was done to minimize order effects.

\section{Data analysis}

In accordance with the majority of research in this area $[1,6,7$, $15,27,31,32]$, the peak lingual pressure from each trial was recorded (in mmHg) and displayed by the instrument. The peak lingual pressure from the 1st second to the 4 th second of each trial (3 seconds) was recorded, and for all participants the peak pressure was observed to be within this time frame of the total four seconds. The values in $\mathrm{mmHg}$ were converted to kilopascal $(\mathrm{kPa})$ by multiplying the recorded value in $\mathrm{mmHg}$ with 0.133 . For each lingual task, the average of peak lingual pressures across the three trials was calculated [1], and this value represented the peak lingual pressure for the MIP lingual task

The principal researcher established intrajudge reliability by re-measuring the dependent measures from the raw data of $15 \%$ of the participants. A second trained rater established interjudge reliability by recording the measures from the raw data of $15 \%$ of the participants. For intra-judge reliability, interclass correlation coefficient was computed for lingual pressures and was established to be $0.99(p<0.01)$. For interjudge reliability, the interclass correlation coefficient for the two sets of measurements for lingual pressures was $0.99(p<0.01)$. Statistical analysis was completed using SPSS v18.0 (SPSS Inc., Chicago, IL). To determine the effects of age (young and old), gender (male and female), and lingual tasks (elevation, protrusion, and depression) on lingual strength, a mixed design 
three-way analysis of variance was adopted. Post hoc analysis was done by applying the Bonferroni correction to account for multiple comparisons.

\section{RESULTS}

\section{Effects of age and gender}

For the MIP tasks, younger adults ( $M=40.80 \mathrm{kPa}, S E=2.36)$ exhibited significantly higher mean peak lingual pressure than the older group $(M=33.66 \mathrm{kPa}, S E=2.41 ; F(1,45)=4.49$,

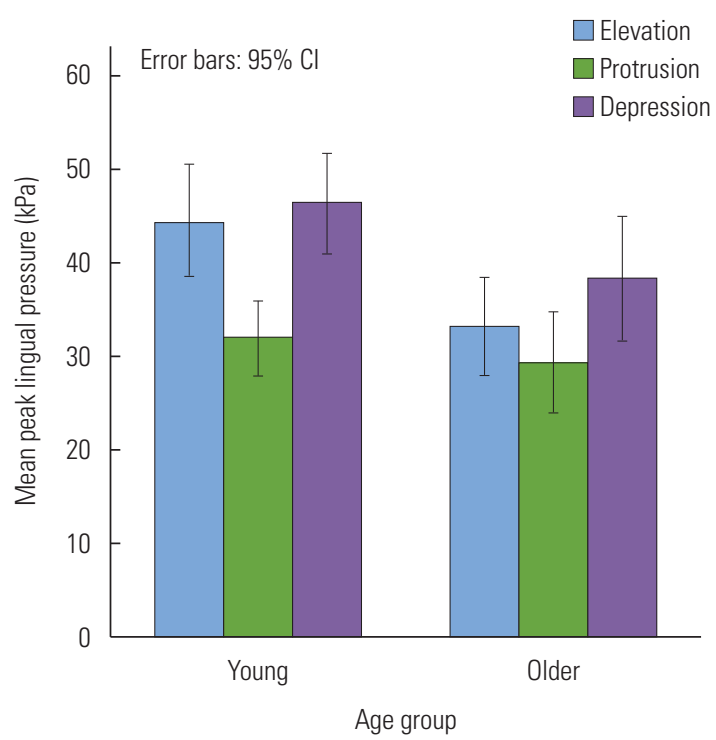

Figure 1. Mean peak lingual pressures (in $\mathrm{kPa}$ ) during the three maximum isometric pressure lingual tasks in young and older healthy adults.

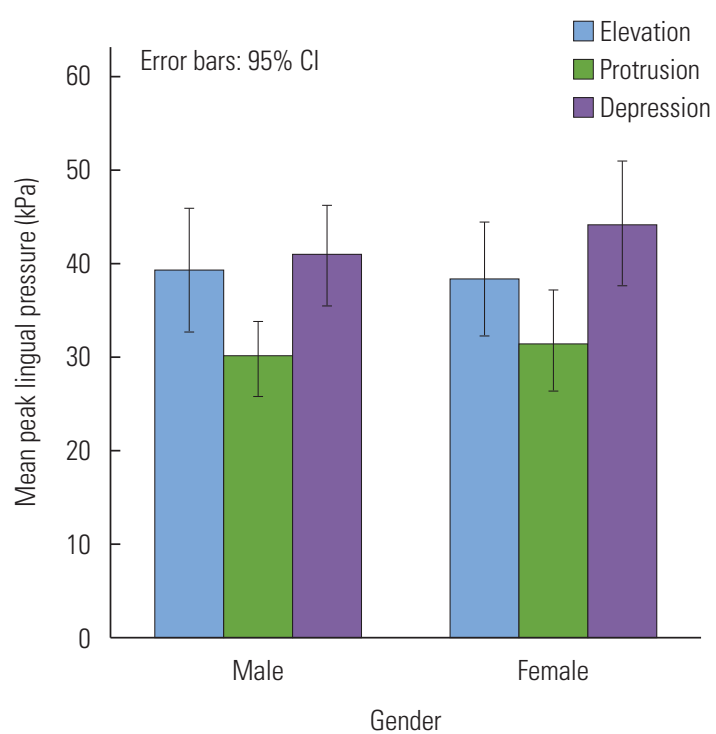

Figure 2. Mean peak lingual pressures (in $\mathrm{kPa}$ ) during the three maximum isometric pressure lingual tasks in men and women. $p=0.04$, partial $\left.\eta^{2}=0.09\right)$. However, there was no significant difference in mean peak lingual pressure between men $(M=$ 36.54, $S E=2.36)$ and women $(M=37.92, S E=2.41 ; F(1,45)=$ $0.17, p=0.68$, partial $\left.\eta^{2}=0.004\right)$. The interaction between age and gender was also not significant $(F(1,45)=2.44, p=0.13$, partial $\eta^{2}=0.05$ ). Figures 1 and 2 illustrates the differences in mean peak lingual pressures between the two age groups and between men and women during the three MIP lingual tasks, respectively.

\section{Effects of lingual task}

There was a significant difference in mean peak lingual pressure during the three MIP lingual tasks, $F(2,90)=33.19$, $p<0.01$, partial $\eta^{2}=0.42$. Post-hoc analysis revealed that mean peak pressure during lingual elevation was significantly greater than that during lingual protrusion, $t(48)=4.83$, $p<0.01$. Mean peak lingual pressures during the lingual protrusion task was significantly lower than that during the lingual depression task, $t(48)=-8.42, p<0.01$. However, there was no significant difference between peak lingual pressures during the lingual elevation and lingual depression tasks, $t(48)=$ $-2.18, p=0.03$. Figure 3 displays the mean peak lingual pressures for the three lingual tasks.

There was a significant interaction between age and lingual task, $F(2,90)=4.28, p=0.02$, partial $\eta^{2}=0.09$. It was established that during lingual elevation, the young adults $(M=44.45 \mathrm{kPa}$, $S E=2.93)$ exhibited higher peak lingual pressures than the older adults ( $M=33.26 \mathrm{kPa}, S E=2.57), t(47)=2.86, p<0.01$. There were no significant differences in mean peak lingual

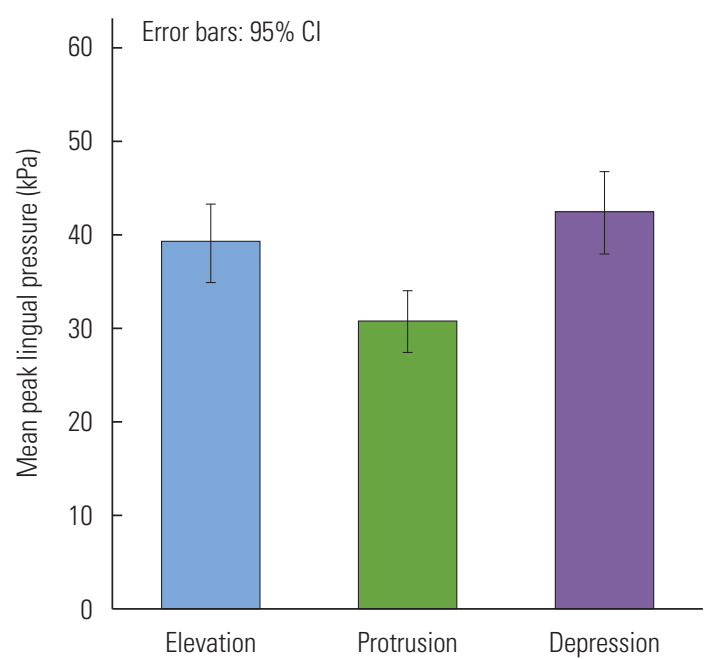

Figure 3. Mean peak lingual pressure (in $\mathrm{kPa}$ ) during the three maximum isometric pressure lingual tasks. 
pressures between the young and older adults for lingual protrusion $\left(M_{y}=31.98 \mathrm{kPa}, S E=1.98 ; M_{o}=29.40 \mathrm{kPa}, S E=2.66\right)$ and lingual depression $\left(M_{y}=46.20 \mathrm{kPa}, S E=2.61 ; M_{o}=38.31\right.$ $\mathrm{kPa}, S E=3.25), t(47)=0.78, p=0.44$, and $t(47)=1.90, p=0.06$, respectively. The interaction between gender and lingual task was not significant, $F(2,90)=0.91, p=0.41$, partial $\eta^{2}=0.02$. However, the triple interaction between age, gender, and lingual task was significant, $F(2,90)=3.88, p=0.02$, partial $\Pi^{2}=0.08$.

\section{DISCUSSION}

This study examined the effects of age and gender during three intraoral lingual tasks (elevation, protrusion, and depression) on peak lingual pressure in healthy adults. The results of this study confirmed the hypothesis that during MIP lingual tasks, young healthy adults exhibited significantly higher peak lingual pressures than older healthy adults. These differences in peak lingual pressures generated by the anterior tongue between the two groups may be attributed to the changes in lingual muscle function with age [4-6,21,26,27,33, 34]. This study also examined the possibility of discussing peak lingual pressures that would be suggestive of reduced lingual strength in healthy nondysphagic individuals. Clark et al. [1] proposed that tongue strength of $20 \mathrm{kPa}$ would be specific in identifying oral dysphagia, on the basis that the anterior tongue in healthy adults can generate pressures of $20 \mathrm{kPa}$ during dry saliva swallows, which was supported by Robbins et al. [5]. This was supported by the results of this study, as both young and older healthy participants could produce peak lingual pressures greater than $20 \mathrm{kPa}$ during MIP lingual tasks. In their study, Clark et al. [1] also reported that individuals with oral dysphagia exhibited average peak lingual pressures of approximately $20 \mathrm{kPa}$. Stierwalt and Youmans [11] observed peak lingual pressures of 55.01 and $33.55 \mathrm{kPa}$ in older healthy individuals and in older individuals with oral dysphagia, respectively. Individuals defined as having "definite dysphagia" exhibited reduced peak lingual pressures of approximately $10.7 \mathrm{kPa}$ during maximum voluntary contraction tasks [35, p. 62]. Stroke patients with dysphagia exhibited mean peak lingual pressures of $35.6 \mathrm{kPa}$ during MIP trials [17]. Thus, considering the range of peak lingual pressures in healthy individuals and in patients with dysphagia from previous research and this study, perhaps peak lingual pressures between 20 to $25 \mathrm{kPa}$ during MIP lingual tasks could serve as a "red flag." Peak lingual pressures within this range could in- dicate reduced lingual strength reserve in older individuals who may not yet overtly exhibit symptoms of oral dysphagia. It may also serve as an indicator of the need to implement LSE in these individuals, which are known to improve lingual strength and reverse the effects of aging on lingual muscle function [12].

In this study, the mean peak lingual pressure in the younger group was $40.80 \mathrm{kPa}$ and that in the older group was 33.66 $\mathrm{kPa}$, which is lower than that reported in other studies $[4,6$, $11,12,24]$. The lower values of peak lingual pressures in both the groups may be attributed to monitoring and maintaining masseter activity during the MIP lingual tasks to below $4.5 \mu \mathrm{V}$ and using a bite block of $2 \mathrm{~mm}$ height, which was used to minimize the influence of jaw muscles on lingual strength. Solomon and Munson [36] have observed that the lingual pressures were highest when no or a very small bite block (2 mm height) was used during intraoral lingual elevation tasks. Perhaps, ensuring low levels of masseter activation by using sEMG, specifying instructions to the participant, and by using a small bite block may indeed influence the "recruitment patterns" [9, p. 833] of the muscles in the oral cavity. While measuring lingual pressures during such lingual tasks and swallowing, interaction and overlap of lingual and jaw activity is to be expected [9], and thus, attempting to compartmentalize and independently measure functions of structures could impede the measurement of true potentials. However, the difference between the mean peak lingual pressure and between the two age groups in this study is consistent with the 10 to 15 $\mathrm{kPa}$ difference that has identified as being "clinically significant" $[27$, p. 7]

There were no significant differences in peak lingual strength during the MIP lingual tasks between men and women. Although, it was hypothesized that greater biological strength in men would result in greater peak lingual pressures $[6,11,15$, 25], similar findings as obtained in this study have also been reported $[7,24,27]$. The differences in mean peak lingual pressures between the two groups were minimal (1.38 kPa). This study also compared the effects of three intraoral MIP lingual tasks involving anterior tongue movement in three directions: elevation, protrusion, and depression. Previously, MIP tasks involving lingual elevation (lingual-palatal contact) $[4,6,11,15$, $24,25]$, protrusion, and lateralization have been recorded $[22,27]$. This study is the first to examine lingual strength during intraoral MIP lingual depression, which resulted in the highest peak pressures among the three lingual tasks. Intraoral lingual depression involves the downward-movement of 
the apex of the tongue, which is secondary to the contraction of the inferior longitudinal muscle and the hyoglossus [37]. All the participants in this study were easily able to execute this lingual task. Instructions for this lingual movement were simple and straightforward, and the placement of tongue bulb array was easily monitored by landmarks in the oral cavity. Adopting such a lingual task in a well-defined MIP lingual exercise protocol may result in greater gains in lingual strength than other lingual tasks that are routinely adopted. Another distinct advantage in adopting the lingual depression task is that the constant and fixed resistance offered by the mandible to generate lingual pressure, could ensure consistency in placement of the tongue and instrumentation during measurement, and increase the ease of instruction for application for home-based LSE.

As the results indicate, there was a differential effect of lingual task on the mean peak pressures with lowest pressures obtained during lingual protrusion. One of the possible reasons for the lower peak pressures during this lingual task might be due to the nature of the placement of the three-bulb tongue array during this task. During this task, the three-bulb tongue array was placed against a tongue depressor for support between the upper and lower incisors, and further stabilization was provided with the investigator's fingers [22]. Compared to the resistance offered during lingual elevation and depression, which was fixed and constant, that during lingual protrusion would have been more varied and less consistent. It was also established that the only significant interaction between age and lingual task in this study was for the lingual elevation condition. For this task, peak lingual pressures in the young group were at least $10 \mathrm{kPa}$ higher than that of the older group, which can be considered to be clinically significant [27]. However, the differences between the two age groups were less than $10 \mathrm{kPa}$ for lingual protrusion and depression.

As previously mentioned, the maximum isometric task involving lingual elevation to the hard palate has been extensively studied. However, limited studies have examined tasks that involve lingual movements in other directions in the oral cavity [27]. In addition, estimating tongue strength using a combination of lingual tasks, such as lingual elevation, protrusion, and lateralization, may be a better estimate of oral lingual strength than when using lingual elevation in isolation [1]. This study only examined anterior MIP lingual tasks. MIP tasks involving the middle and posterior tongue and lateral lingual movements may highlight differential effects of lingual tasks on generation of peak lingual pressures.

The lingual muscles have a major role in the oral and pharyngeal stages of swallowing [2]. During the MIP tasks, the lingual muscles are involved in isometric contraction and exertion of maximal pressure against a constant resistance. Such tasks involving different intraoral lingual movements are those that can be routinely adopted clinically, especially when oral dysphagia is secondary to lingual muscle weakness. Clinically, the advantages of the lingual depression task should be taken into consideration, such as ease of completion, simple instructions and feedback, consistent resistance, and higher peak lingual pressures than other tasks such as protrusion. Thus, it would be worthwhile to clinically implement such an exercise paradigm incorporating this novel lingual task and compare gains in lingual strength to that recorded with other lingual movements. Studying the MIP lingual tasks adopted in this study in a LSE program may also help in determining the ideal combination of lingual tasks that would offer the most benefit to individuals with dysphagia by improving their lingual strength. Lingual strengthening exercises are gaining popularity in the treatment of patients with dysphagia, and target muscles that are readily accessible within the swallowing mechanism [21].

\section{REFERENCES}

1. Clark HM, Henson PA, Barber WD, Stierwalt JAG, Sherrill M. Relationships among subjective and objective measures of tongue strength and oral phase swallowing impairments. Am J Speech Lang Pathol. 2003;12:40-50.

2. Dodds WJ. The physiology of swallowing. Dysphagia. 1989;3:171178.

3. Steele CM, Bailey GL, Molfenter SM, Yeates EM. Rationale for strength and skill goals in tongue resistance training: a review. Perspect ASHA Spec Interest Groups. 2009;18:49-54.

4. Fei T, Polacco RC, Hori SE, Molfenter SM, Peladeau-Pigeon M, Tsang C, et al. Age-related differences in tongue-palate pressures for strength and swallowing tasks. Dysphagia. 2013;28:575-581.

5. Robbins JA, Levine R, Wood J, Roecker EB, Luschei E. Age effects on lingual pressure generation as a risk factor for dysphagia. J. Gerontol. 1995;50A:M257-M262.

6. Youmans SR, Stierwalt JAG. Measures of tongue function related to normal swallowing. Dysphagia. 2006;21:102-111.

7. Youmans SR, Youmans GL, Stierwalt JAG. Differences in tongue strength across age and gender: is there a diminished strength reserve? Dysphagia. 2009;24:57-65.

8. Clark HM. Specificity of training in lingual musculature. J Speech Lang Hear Res. 2012;55:657-667.

9. Palmer PM, Jaffe DM, McCulloch TM, Finnegan EM, Van Daele 
DJ, Luschei ES. quantitative contributions of the muscles of the tongue, floor-of- mouth, jaw, and velum to tongue-to-palate pressure generation. J Speech Lang Hear Res. 2008;51:828-835.

10. Logemann JA. Evaluation and treatment of swallowing disorders. Austin, TX: PRO-ED; 1998.

11. Stierwalt JAG, Youmans SR. Tongue measures in individuals with normal and impaired swallowing. Am J Speech Lang Pathol. 2007; 16:148-156.

12. Robbins JA, Gangnon RE, Theis SM, Kays SA, Hewitt AL, Hind JA. The effects of lingual exercise on swallowing in older adults. J Am Geriatr Soc. 2005;53:1483-1489.

13. McArdle WD, Katch FI, Katch VL. Essentials of exercise physiology (3rd ed.). Baltimore, MD: Lippincott Williams \& Wilkins; 2006.

14. Adams V, Mathisen B, Baines S, Lazarus C, Callister R. A systematic review and meta-analysis of measurements of tongue and hand strength and endurance using the Iowa Oral Performance Instrument (IOPI). Dysphagia. 2013;28:350-369.

15. Gingrich LL, Stierwalt JA, Hageman CF, LaPointe LL. Lingual propulsive pressures across consistencies generated by the anteromedian and posteromedian tongue by healthy young adults. J Speech Lang Hear Res. 2012;55:960-972.

16. Oh J. Effects of tongue strength training and detraining on tongue pressures in healthy adults. Dysphagia. 2015;30:315-320.

17. Robbins JA, Kays SA, Gangnon RE, Hind JA, Hewitt AL, Gentry LR, et al. The effects of lingual exercise in stroke patients with dysphagia. Arch Phys Med Rehabil. 2007;88:150-158.

18. Steele CM, Bailey GL, Polacco REC, Hori SF, Molfenter SM, Oshalla $\mathrm{M}$, et al. Outcomes of tongue-pressure strength and accuracy training for dysphagia following acquired brain injury. Int J Speech Lang Pathol. 2013;15:492-502.

19. Clark H, Shelton N. Training effects of the effortful swallow under three exercise conditions. Dysphagia. 2014;29:553-563.

20. Yeates EM, Molfenter SM, Steele CM. Improvements in tongue strength and pressure-generation precision following a tonguepressure training protocol in older individuals with dysphagia: three case reports. Clin Interv Aging. 2008;3:735-747.

21. McKenna VS, Zhang B, Haines MB, Kelchner LN. A systematic review of isometric lingual strength-training programs in adults with and without dysphagia. Am J Speech Lang Pathol. 2017;26: 524-539.

22. Clark HM, O'Brien K, Calleja A, Corrie SN. Effects of directional exercise on lingual strength. J Speech Lang Hear Res. 2009;52:
1034-1047.

23. Robbins J. Oral strengthening and swallowing outcomes. Perspect ASHA Spec Interest Groups. 2003;12:16-20.

24. Nicosia MA, Hind JA, Roecker EB, Carnes M, Doyle J, Dengel GA, et al. Age effects on the temporal evolution of isometric and swallowing pressure. J Gerontol. 2000;55A:M634-M640.

25. Vanderwegen J, Guns C, Van Nuffelen G, Elen R, De Bodt M. The influence of age, sex, bulb position, visual feedback, and the order of testing on maximum anterior and posterior tongue strength and endurance in healthy Belgian adults. Dysphagia. 2013;28:159-166.

26. Robbins J, Humpal NS, Banaszynski K, Hind J, Rogus-Pulia N. Agerelated differences in pressures generated during isometric presses and swallows by healthy adults. Dysphagia. 2016;31:90-93.

27. Clark HM, Solomon NP. Age and sex differences in orofacial strength. Dysphagia. 2012;27:2-9.

28. Mortimore IL, Fiddes P, Stephens S, Douglas NJ. Tongue protrusion force and fatigability among male and female subjects. Eur Respir J. 1999;14:191-195.

29. Kay Elemetrics Corp. Instruction manual: Digital swallowing workstation Model 7200. Lincoln Park, NJ: Kay Elemetrics; 2004.

30. Vaiman M, Eviatar E, Segal S. Surface electromyographic studies of swallowing in normal subjects: a review of 440 adults. Report 2. Otolaryngol Head Neck Surg. 2004;131:773-780.

31. Lazarus C, Logemann JA, Huang CF, Rademaker AW. Effects of two types of tongue strengthening exercises in young normals. Folia Phoniatr Logop. 2003;55:199-205.

32. Robin DA, Goel A, Somodi LB, Luschei ES. Tongue strength and endurance: relation to highly skilled movements. J Speech Lang Hear Res. 1992;35:1239-1245.

33. Steele C. Optimal approaches for measuring tongue-pressure functional reserve. J Aging Res. 2013.

34. Todd JT, Lintzenich CR, Butler SG. Isometric and swallowing tongue strength in healthy adults. The Laryngoscope. 2013;123: 2469-2473.

35. Yoshida M, Kikutani T, Tsuga K, Utanohara Y, Hayashi R, Akagawa Y. Decreased tongue pressure reflects symptom of dysphagia. Dysphagia. 2006;21:61-65.

36. Solomon NP, Munson B. The effect of jaw position on measures of tongue strength and endurance. J Speech Lang Hear Res. 2004;47: 584-594.

37. Drake RL, Vogl W, Mitchell AWM. Gray's anatomy for students. Philadelphia, PA: Churchill Livingstone, Elsevier; 2005. 